\title{
DYNAMIC FORCE ANALYSIS OF A SIX-LINK PLANAR MECHANISM UNDER CONSIDERATION OF FRICTION AT THE JOINTS
}

\author{
NGUYen Phong DiEn AND NGUYen VAN KHANG \\ Hanoi University of Technology
}

\begin{abstract}
The dynamic analysis of mechanisms with joints friction is complex since the frictional force depends nonlinearly on the resultant reactive force between the two mating surfaces of the joint. In this paper a non-iterative approximate method is used for determining the joint reaction forces and the driving torque of mechanisms is considered. By using the computing program MATLAB the dynamic forces of a six-link planar mechanism are calculated with this method.
\end{abstract}

\section{Introduction}

Improvements in the mechanical characteristics of machines consist typically of trends to enhance their productivity by increasing the operational speed. In mechanism that operates at high speeds, dynamic forces are often greater than static or other externally applied forces and play an important role in the design, sizing of links and bearings.

The dynamic analysis of a mechanism reveals all the joint reaction forces, including the driving torque required to generate a prescribed steady state motion. It is well known that by application of D'Alembert's principle, a dynamic problem can be converted into a static problem. So, every member of a mechanism can be considered to be in equilibrium under the combined effect of all the externally applied forces, the joint reaction forces and its own resultant inertia force and moment. By neglecting frictional forces at the joints, the joint reaction forces can be easily calculated from the dynamic equilibrium equations, which are linear algebraic equations $[1,2,5]$.

The dynamic analysis of mechanisms with friction at the joints is complex since the frictional force depends nonlinearly on the resultant reactive force between the two mating surfaces of the joint. These joint reaction forces depend again on the external loading and on the motion of the mechanism. A solution with reasonable accuracy can be obtained and undue complications is avoided by using a non-iterative approximate method developed by Funk and Kong [3]. . In this paper, the non-iterative method is applied to determine the joint reaction forces and the driving torque of a six-link planar mechanism with friction. A specialized code has been developed in the high-level programming language MATLAB for this study. 


\section{Mathematical model and calculating method}

In hinged joints, the friction occurs between the pin and the inside cylindrical surface of the hole in the connected link. The frictional force acts in a direction perpendicular to the normal contact force and will apply a frictional torque to the joint [4]:

$$
M_{(i, j)}^{R}=\operatorname{sign}\left(\dot{\varphi}_{i}-\dot{\varphi}_{j}\right) r_{(i, j)} \mu_{(i, j)}^{\gamma} G_{(i, j)}^{R},
$$

where $M_{(i, j)}^{R}$ is the frictional torque acting at the joint $(i, j)$ between $i$-th and $j$-th links, $r_{(i, j)}$ is the pin radius of the joint, $\dot{\varphi}_{i}$ and $\dot{\varphi}_{j}$ are the angular velocities of the $i$-th and $j$-th links respectively. The coefficient of friction $\mu_{(i, j)}^{\gamma}$ is normally larger than the dynamic coefficient of the sliding friction $\mu_{(i, j)}$, which can be experimentally determined as (see $[3])$ :

$$
\mu_{(i, j)}^{\gamma} \approx(1, \ldots, 1.57) \mu_{(i, j)} .
$$

$G_{(i, j)}^{R}$ denotes the joint reaction force exerted on the $i$-th link by the $j$-th link and may be resolved into $x$ - and $y$-components:

$$
G_{(i, j)}^{R}=\sqrt{\left(G_{(i, j) x}^{R}\right)^{2}+\left(G_{(i, j) y}^{R}\right)^{2}} .
$$

Substituting (2.3) in (2.1), the frictional torque can be expressed in terms of $G_{(i, j) x}^{R}$ and $G_{(i, j) y}^{R}$ as

$$
M_{(i, j)}^{R}=\operatorname{sign}\left(\dot{\varphi}_{i}-\dot{\varphi}_{j}\right) r_{(i, j)} \mu_{(i, j)}^{\gamma} \sqrt{\left(G_{(i, j) x}^{R}\right)^{2}+\left(G_{(i, j) y}^{R}\right)^{2}} .
$$

From (2.4), it is seen that the set of equilibrium equations for calculating the joint reaction forces includes nonlinear terms due to the effect of friction. This set of equations can be solved iteratively using the numerical method. However, a complex mechanism with numerous links may involve a large number of equations that require a very large number of iterative calculations until the solution converges. For such cases, it is important to find a solution procedure which is based on non-iterative approximations.

Using the Taylor series expansion the first-order approximation of $G_{(i, j)}^{R}$ from $(2.3)$ is defined by the terms of its Taylor series up to the first derivative as follows

$$
\begin{aligned}
G_{(i, j)}^{R}= & \sqrt{\left(G_{(i, j) x}^{R}\right)^{2}+\left(G_{(i, j) y}^{R}\right)^{2}} \approx G_{(i, j)}+\left(G_{(i, j) x}^{R}-G_{(i, j) x}\right) \frac{G_{(i, j) x}}{G_{(i, j)}} \\
& +\left(G_{(i, j) y}^{R}-G_{(i, j) y}\right) \frac{G_{(i, j) y}}{G_{(i, j)}} \approx \frac{1}{G_{(i, j)}}\left(G_{(i, j) x} G_{(i, j) x}^{R}+G_{(i, j) y} G_{(i, j) y}^{R}\right),
\end{aligned}
$$

where $G_{(i, j)}$ is the joint reaction force when the mechanism is assumed to be free from friction:

$$
G_{(i, j)}=\sqrt{\left(G_{(i, j) x}\right)^{2}+\left(G_{(i, j) y}\right)^{2}} .
$$


Substituting (2.5) in (2.1), the expression for $M_{(i, j)}^{R}$ can be written in the form

$$
\begin{aligned}
M_{(i, j)}^{R} & \approx \operatorname{sign}\left(\dot{\varphi}_{i}-\dot{\varphi}_{j}\right) \frac{r_{(i, j)} \mu_{(i, j)}^{\gamma}}{G_{(i, j)}}\left(G_{(i, j) x} G_{(i, j) x}^{R}+G_{(i, j) y} G_{(i, j) y}^{R}\right) \\
& \approx \delta_{(i, j) x} G_{(i, j) x}^{R}+\delta_{(i, j) y} G_{(i, j) y}^{R},
\end{aligned}
$$

where $\delta_{(i, j) x}$ and $\delta_{(i, j) y}$ are expressed in terms of the joint reaction force $G_{(i, j) x}$ and $G_{(i, j) y}$ as

$$
\begin{aligned}
& \delta_{(i, j) x}=\operatorname{sign}\left(\dot{\varphi}_{i}-\dot{\varphi}_{j}\right) r_{(i, j)} \mu_{(i, j)}^{\gamma} \frac{G_{(i, j) x}}{\sqrt{\left(G_{(i, j) x}\right)^{2}+\left(G_{(i, j) y}\right)^{2}}} \\
& \delta_{(i, j) y}=\operatorname{sign}\left(\dot{\varphi}_{i}-\dot{\varphi}_{j}\right) r_{(i, j)} \mu_{(i, j)}^{\gamma} \frac{G_{(i, j) y}}{\sqrt{\left(G_{(i, j) x}\right)^{2}+\left(G_{(i, j) y}\right)^{2}}}
\end{aligned}
$$

Note that $G_{(i, j) x}$ and $G_{(i, j) y}$ can be determined from the dynamic equilibrium equations of the mechanism without friction at the joints and then, $\delta_{(i, j) x}$ and $\delta_{(i, j) y}$ can be easily calculated by using equations (2.8) and (2.9). When the values of $\delta_{(i, j) x}$ and $\delta_{(i, j) y}$ are known, the frictional torque $M_{(i, j)}^{R}$ and the components $G_{(i, j) x}^{R}, G_{(i, j) y}^{R}$ of the joint reaction force have a linear relation in (2.7) with one another. Finally, the equilibrium equations for joint reaction forces with friction become linear algebraic equations. Hence, this solution procedure can be well called as the non-iterative approximate method.

The nonlinear terms truncated for equation (2.5) will determine the accuracy of the solution. A thorough discussion of the truncation error is given in section 4 .

\section{Dynamic force analysis of a six-link planar mechanism}

Consider a six-link planar mechanism shown in Fig. 1. Seven revolute pairs are required to connect the six links. The crank is termed as link 2 which is hinged to the ground link 1.

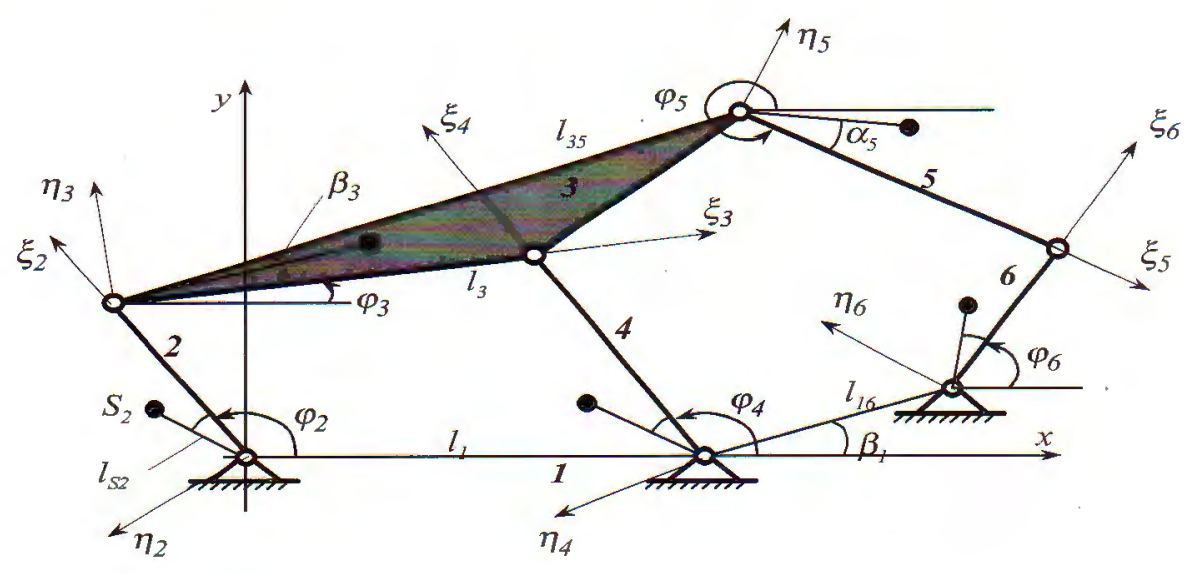

Fig. 1. Kinematic diagram of the six-link planar mechanism 
First, by kinematic analysis, we determine the angular accelerations of the links and the linear accelerations of the center of mass $S_{i}(i=2, \ldots, 6)$. The configuration of the crank is also prescribed by the angle $\varphi_{2}$ and we have to determine the configuration of the other four link expressed by the rotating angles $\varphi_{3}, \varphi_{4}, \varphi_{5}$ and $\varphi_{6}$. The kinematic relationships for the links of the mechanism can be expressed in the form

$$
\begin{aligned}
& l_{2} \cos \varphi_{2}+l_{3} \cos \varphi_{3}-l_{4} \cos \varphi_{4}-l_{1}=0, \\
& l_{2} \sin \varphi_{2}+l_{3} \sin \varphi_{3}-l_{4} \sin \varphi_{4}=0, \\
& l_{2} \cos \varphi_{2}+l_{35} \cos \left(\varphi_{3}+\beta_{3}\right)+l_{5} \cos \varphi_{5}-l_{6} \cos \varphi_{6}-l_{1}-l_{16} \cos \beta_{1}=0, \\
& l_{2} \sin \varphi_{2}+l_{35} \sin \left(\varphi_{3}+\beta_{3}\right)+l_{5} \sin \varphi_{5}-l_{6} \sin \varphi_{6}-l_{16} \sin \beta_{1}=0,
\end{aligned}
$$

where $l_{i}$ denotes the length of the $i$-th link. By differentiating (3.1) with respect to time, the relationships for $\dot{\varphi}_{i}(i=2, \ldots, 6)$ can be written in matrix form as

$$
\left[\begin{array}{cccc}
-l_{3} \sin \varphi_{3} & l_{4} \sin \varphi_{4} & 0 & 0 \\
l_{3} \cos \varphi_{3} & -l_{4} \cos \varphi_{4} & 0 & 0 \\
-l_{35} \sin \left(\varphi_{3}+\beta_{3}\right) & 0 & -l_{5} \sin \varphi_{5} & l_{6} \sin \varphi_{6} \\
l_{35} \cos \left(\varphi_{3}+\beta_{3}\right) & 0 & l_{5} \cos \varphi_{5} & -l_{6} \cos \varphi_{6}
\end{array}\right]\left[\begin{array}{c}
\dot{\varphi}_{3} \\
\dot{\varphi}_{4} \\
\dot{\varphi}_{5} \\
\dot{\varphi}_{6}
\end{array}\right]=\left[\begin{array}{c}
l_{2} \sin \varphi_{2} \\
-l_{2} \cos \varphi_{2} \\
l_{2} \sin \varphi_{2} \\
-l_{2} \cos \varphi_{2}
\end{array}\right] \dot{\varphi}_{2}
$$

Differentiating (3.2) again with respect to time, the relationships for $\ddot{\varphi}_{i}(i=2, \ldots, 6)$ take the form

$$
\begin{aligned}
& {\left[\begin{array}{cccc}
-l_{3} \sin \varphi_{3} & l_{4} \sin \varphi_{4} & 0 & 0 \\
l_{3} \cos \varphi_{3} & -l_{4} \cos \varphi_{4} & 0 & 0 \\
-l_{35} \sin \left(\varphi_{3}+\beta_{3}\right) & 0 & -l_{5} \sin \varphi_{5} & l_{6} \sin \varphi_{6} \\
l_{35} \cos \left(\varphi_{3}+\beta_{3}\right) & 0 & l_{5} \cos \varphi_{5} & -l_{6} \cos \varphi_{6}
\end{array}\right]\left[\begin{array}{c}
\ddot{\varphi}_{3} \\
\ddot{\varphi}_{4} \\
\ddot{\varphi}_{5} \\
\ddot{\varphi}_{6}
\end{array}\right]=} \\
& {\left[\begin{array}{c}
l_{2} \ddot{\varphi}_{2} \sin \varphi_{2}+l_{2} \dot{\varphi}_{2}^{2} \cos \varphi_{2}+l_{3} \dot{\varphi}_{3}^{2} \cos \varphi_{3}-l_{4} \dot{\varphi}_{4}^{2} \cos \varphi_{4} \\
-l_{2} \ddot{\varphi}_{2} \cos \varphi_{2}+l_{2} \dot{\varphi}_{2}^{2} \sin \varphi_{2}+l_{3} \dot{\varphi}_{3}^{2} \sin \varphi_{3}-l_{4} \dot{\varphi}_{4}^{2} \sin \varphi_{4} \\
l_{2} \ddot{\varphi}_{2} \sin \varphi_{2}+l_{2} \dot{\varphi}_{2}^{2} \cos \varphi_{2}+l_{35} \dot{\varphi}_{3}^{2} \cos \left(\varphi_{3}+\beta_{3}\right)+l_{5} \dot{\varphi}_{3}^{2} \cos \varphi_{5}-l_{6} \dot{\varphi}_{6}^{2} \cos \varphi_{6} \\
-l_{2} \ddot{\varphi}_{2} \cos \varphi_{2}+l_{2} \dot{\varphi}_{2}^{2} \sin \varphi_{2}+l_{35} \dot{\varphi}_{3}^{2} \sin \left(\varphi_{3}+\beta_{3}\right)+l_{5} \dot{\varphi}_{3}^{2} \sin \varphi_{5}-l_{6} \dot{\varphi}_{6}^{2} \cos \varphi_{6}
\end{array}\right] .}
\end{aligned}
$$

Thus, the rotating angles, angular velocities and angular accelerations of the links can be calculated from equations (3.1), (3.2) and (3.3). The position of the center of mass $S_{i}$ in the fixed coordinate frame $\{x, y\}$ is given by

$$
\left[\begin{array}{l}
x_{S i} \\
y_{S i}
\end{array}\right]=\left[\begin{array}{l}
x_{0 i} \\
y_{0 i}
\end{array}\right]+\mathbf{A}_{i}\left[\begin{array}{c}
\xi_{S i} \\
\eta_{S i}
\end{array}\right]
$$

where $\xi_{S i}, \eta_{S i}$ are coordinates of $S_{i}$ in the moving coordinate frame $\left\{\xi_{i}, \eta_{i}\right\}$ as shown in Fig. 1:

$$
\xi_{S i}=l_{S i} \cos \alpha_{i}, \quad \eta_{S i}=l_{S i} \sin \alpha_{i}, \quad(i=2, \ldots, 6),
$$

and $x_{0 i}, y_{0 i}$ are coordinates of the origin of the frame $\left\{\xi_{i}, \eta_{i}\right\}$ in the fixed frame $\{x, y\}$. The matrix $\mathbf{A}_{i}$ is defined by

$$
\mathbf{A}_{i}=\left[\begin{array}{cc}
\cos \varphi_{i} & -\sin \varphi_{i} \\
\sin \varphi_{i} & \cos \varphi_{i}
\end{array}\right]
$$


Hence, the components of the acceleration of $S_{i}$ can be derived by the second derivative of equation (3.4) with respect to time (see also [6]):

$$
\left[\begin{array}{c}
\ddot{x}_{S i} \\
\ddot{y}_{S i}
\end{array}\right]=\left[\begin{array}{c}
\ddot{x}_{0 i} \\
\ddot{x}_{0 i}
\end{array}\right]+\left(\ddot{\varphi}_{i} \mathbf{I}-\dot{\varphi}_{i}^{2} \mathbf{E}\right) \mathbf{A}_{i}\left[\begin{array}{c}
\xi_{S i} \\
\eta_{S i}
\end{array}\right], \quad(i=2, \ldots, 6),
$$

where

$$
\mathbf{I}=\left[\begin{array}{cc}
0 & -1 \\
1 & 0
\end{array}\right], \quad \mathbf{E}=\left[\begin{array}{ll}
0 & 1 \\
1 & 0
\end{array}\right]
$$

The next step will be the formulation of the dynamic equilibrium equations for the $i$-th link of the mechanism. The link is subjected to its gravitational force $P_{i}$. The inertia force and the inertia torque are known as follow

$$
F_{i x}^{q t}=m_{i} \ddot{x}_{S i}, \quad F_{i y}^{q t}=m_{i} \ddot{y}_{S i}, \quad M_{i}^{q t}=J_{S i} \ddot{\varphi}_{i}
$$

where $m_{i}$ is the mass of the $i$-th link and $J_{S i}$ represents the moment of inertia of the link about the axis passing through the center of mass $S_{i}$ and perpendicular to the plane of motion. Fig. 2 shows an exploded view of the mechanism with the free-body diagrams of each links, where $G_{(i, j)}^{R}=G_{(j, i)}^{R}, M_{(i, j)}^{R}=M_{(j, i)}^{R}$. A driving torque $M_{d}$ applied on the crank 2 and a loading torque $M_{6}$ acts on the output link 6 .

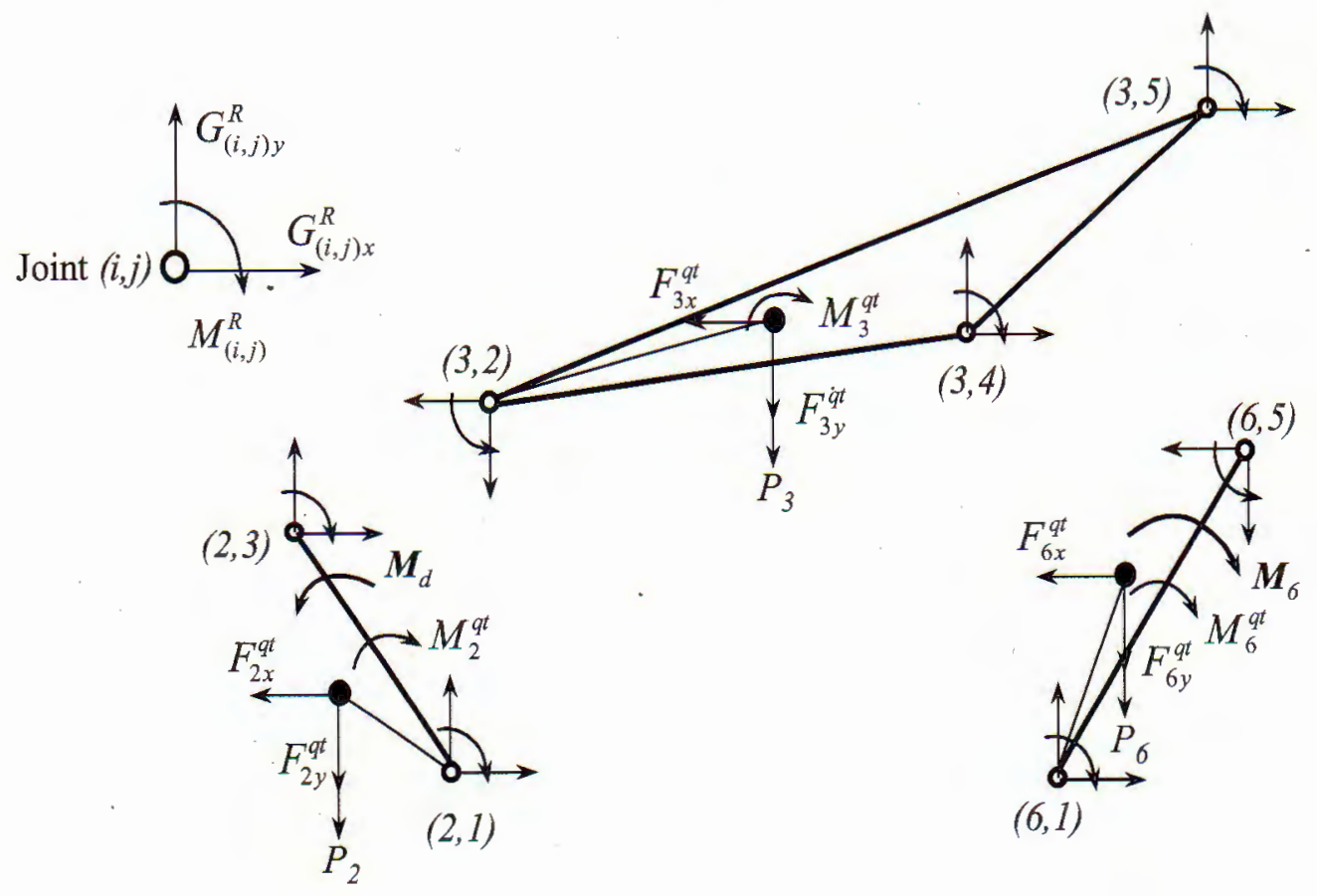

Fig. 2. Free-body diagrams for links 2,3 and 6

By using D'Alembert's principle, dynamic equilibrium equations for each link are established. Combining these equations yields 


$$
\begin{aligned}
& G_{(2,1) x}^{R}+G_{(2,3) x}^{R}-F_{2 x}^{q t}=0 \\
& G_{(2,1) y}^{R}+G_{(2,3) y}^{R}-F_{2 y}^{q t}-P_{2}=0
\end{aligned}
$$

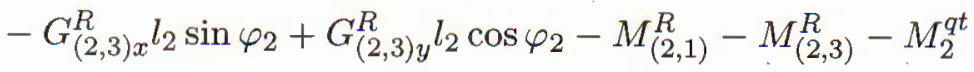

$$
\begin{aligned}
& +F_{2 x}^{q t} l_{S 2} \sin \left(\varphi_{2}+\alpha_{2}\right)-F_{2 y}^{q t} l_{S 2} \cos \left(\varphi_{2}+\alpha_{2}\right)-P_{2} l_{S 2} \cos \left(\varphi_{2}+\alpha_{2}\right)+M_{d}=0 \text {, } \\
& G_{(3,4) x}^{R}+G_{(3,5) x}^{R}-G_{(3,2) x}^{R}-F_{3 x}^{q t}=0, \\
& G_{(3,4) y}^{R}+G_{(3,5) y}^{R}-G_{(3,2) y}^{R}-F_{3 y}^{q t}-P_{3}=0, \\
& -G_{(3,4) x}^{R} l_{3} \sin \varphi_{3}+G_{(3,4) y}^{R} l_{2} \cos \varphi_{3}-G_{(3,5) x}^{R} l_{35} \sin \left(\varphi_{3}+\beta_{3}\right) \\
& +G_{(3,5) y}^{R} l_{35} \cos \left(\varphi_{3}+\beta_{3}\right)+M_{(3,2)}^{R}-M_{(3,4)}^{R}-M_{(3,5)}^{R}-M_{3}^{q t} \\
& +F_{3 x}^{q t} l_{S 3} \sin \left(\varphi_{3}+\alpha_{3}\right)-F_{3 y}^{q t} l_{S 3} \cos \left(\varphi_{3}+\alpha_{3}\right)-P_{3} l_{S 3} \cos \left(\varphi_{3}+\alpha_{3}\right)=0 \text {, } \\
& G_{(4,1) x}^{R}-G_{(4,3) x}^{R}-F_{4 x}^{q t}=0, \\
& G_{(4,1) y}^{R}-G_{(4,3) y}^{R}-F_{4 y}^{q t}-P_{4}=0, \\
& G_{(4,3) x^{2}}^{R} \sin \varphi_{4}-G_{(4,3) y}^{R} l_{4} \cos \varphi_{4}-M_{(4,1)}^{R}+M_{(4,3)}^{R}-M_{4}^{q t} \\
& +F_{4 x}^{q t} l_{S 4} \sin \left(\varphi_{4}+\alpha_{4}\right)-F_{4 y}^{q t} l_{S 4} \cos \left(\varphi_{4}+\alpha_{4}\right)-P_{4} l_{S 4} \cos \left(\varphi_{4}+\alpha_{4}\right)=0 \text {, } \\
& G_{(5,6) x}^{R}-G_{(5,3) x}^{R}-F_{5 x}^{q t}=0, \\
& G_{(5,6) y}^{R}-G_{(5,3) y}^{R}-F_{5 y}^{q t}-P_{5}=0 \\
& -G_{(5,6) x}^{R} l_{5} \sin \varphi_{5}+G_{(5,6) y}^{R} l_{5} \cos \varphi_{5}+M_{(5,3)}^{R}-M_{(5,6)}^{R}-M_{5}^{q t} \\
& +F_{5 x}^{q t} l_{S 5} \sin \left(\varphi_{5}+\alpha_{5}\right)-F_{5 y}^{q t} l_{S 5} \cos \left(\varphi_{5}+\alpha_{5}\right)-P_{5} l_{S 5} \cos \left(\varphi_{5}+\alpha_{5}\right)=0 \text {, } \\
& G_{(6,1) x}^{R}-G_{(6,5) x}^{R}-F_{6 x}^{q t}=0, \\
& G_{(6,1) y}^{R}-G_{(6,5) y}^{R}-F_{6 y}^{q t}-P_{6}=0 \text {, } \\
& G_{(6,5) x}^{R} l_{6} \sin \varphi_{6}-G_{(6,5) y_{,}}^{R} l_{6} \cos \varphi_{6}+M_{(6,5)}^{R}-M_{(6,1)}^{R}-M_{6}^{q t} \\
& +F_{6 x}^{q t} l_{S 6} \sin \left(\varphi_{6}+\alpha_{6}\right)-F_{6 y}^{q t} l_{S 6} \cos \left(\varphi_{6}+\alpha_{6}\right)-P_{6} l_{S 6} \cos \left(\varphi_{6}+\alpha_{6}\right)=0 \text {, }
\end{aligned}
$$

By solving these fifteen equations, we can determine the driving torque $M_{d}$ and fourteen components of the joint reaction forces. With the help of equation (2.7), the equilibrium equations for the complete mechanism can be written in the form

$$
\mathrm{D} \cdot \mathbf{g}=\mathbf{b}
$$

where 
$\mathbf{D}=\left[\begin{array}{ccccccc}1 & 0 & 1 & 0 & 0 & 0 & 0 \\ 0 & 1 & 0 & 1 & 0 & 0 & 0 \\ -\delta_{(2,1) x} & -\delta_{(2,1) y} & -y_{2}-\delta_{(2,3) x} & x_{2}-\delta_{(2,3) y} & 0 & 0 & 0 \\ 0 & 0 & -1 & 0 & 1 & 0 & 1 \\ 0 & 0 & 0 & -1 & 0 & 1 & 0 \\ 0 & 0 & \delta_{(3,2) x} & \delta_{(3,2) y} & -y_{3}-\delta_{(3,4) y} & x_{3}-\delta_{(4,3) y} & y_{35}-\delta_{(3,5) x} \\ 0 & 0 & 0 & 0 & -1 & 0 & 0 \\ 0 & 0 & 0 & 0 & 0 & -1 & 0 \\ 0 & 0 & 0 & 0 & y_{4}+\delta_{(4,3) x} & x_{4}+\delta_{(4,3) y} & 0 \\ 0 & 0 & 0 & 0 & 0 & 0 & -1 \\ 0 & 0 & 0 & 0 & 0 & 0 & 0 \\ 0 & 0 & 0 & 0 & 0 & 0 & \delta_{(5,3) x} \\ 0 & 0 & 0 & 0 & 0 & 0 & 0 \\ 0 & 0 & 0 & 0 & 0 & 0 & 0 \\ 0 & 0 & 0 & 0 & 0 & 0 & 0\end{array}\right.$

$\left.\begin{array}{cccccccc}0 & 0 & 0 & 0 & 0 & 0 & 0 & 0 \\ 0 & 0 & 0 & 0 & 0 & 0 & 0 & 0 \\ 0 & 0 & 0 & 0 & 0 & 0 & 0 & 1 \\ 0 & 0 & 0 & 0 & 0 & 0 & 0 & 0 \\ 1 & 0 & 0 & 0 & 0 & 0 & 0 & 0 \\ x_{35}-\delta_{(3,5) y} & 0 & 0 & 0 & 0 & 0 & 0 & 0 \\ 0 & 1 & 0 & 0 & 0 & 0 & 0 & 0 \\ 0 & 0 & 1 & 0 & 0 & 0 & 0 & 0 \\ 0 & -\delta_{(4,1) x} & -\delta_{(4,1) y} & 0 & 0 & 0 & 0 & 0 \\ 0 & 0 & 0 & 1 & 0 & 0 & 0 & 0 \\ -1 & 0 & 0 & 0 & 1 & 0 & 0 & -0 \\ \delta_{(5,3) y} & 0 & 0 & -y_{5}-\delta_{(5,6) x} & x_{5}-\delta_{(5,6) y} & 0 & 0 & 0 \\ 0 & 0 & 0 & -1 & 0 & 1 & 0 & 0 \\ 0 & 0 & 0 & 0 & -1 & 0 & 1 & 0 \\ 0 & 0 & 0 & y_{6}+\delta_{(6,5) x} & -x_{6}+\delta_{(6,5) y} & -\delta_{(6,1) x} & -\delta_{(6,1) y} & 0\end{array}\right]$,




$$
\mathbf{g}=\left[\begin{array}{c}
G_{(2,1) x}^{R} \\
G_{(2,1) y}^{R} \\
G_{(3,2) x}^{R} \\
G_{(3,2) y}^{R} \\
G_{(3,4) x}^{R} \\
G_{(3,4) y}^{R} \\
G_{(3,5) x}^{R} \\
G_{(3,5) y}^{R} \\
G_{(4,1) x}^{R} \\
G_{(4,1) y}^{R} \\
G_{(5,6) x}^{R} \\
G_{(5,6) y}^{R} \\
G_{(6,1) x}^{R} \\
G_{(6,1) y}^{R} \\
M_{d}^{R}
\end{array}\right], \quad \mathbf{b}=\left[\begin{array}{c}
F_{2 x}^{q t} \\
F_{2 y}^{q t}+P_{2} \\
M_{2}^{q t}-F_{2 x}^{q t} l_{S 2} \sin \left(\varphi_{2}+\alpha_{2}\right)+\left(F_{2 y}^{q t}+P_{2}\right) l_{S 2} \cos \left(\varphi_{2}+\alpha_{2}\right) \\
F_{3 x}^{q t} \\
F_{3 y}^{q t}+P_{3} \\
M_{3}^{q t}-F_{3 x}^{q t} l_{S 3} \sin \left(\varphi_{3}+\alpha_{3}\right)+\left(F_{3 y}^{q t}+P_{3}\right) l_{S 3} \cos \left(\varphi_{3}+\alpha_{3}\right) \\
F_{4 x}^{q t} \\
F_{4 y}^{q t}+P_{4} \\
M_{4}^{q t}-F_{4 x}^{q t} l_{S 4} \sin \left(\varphi_{4}+\alpha_{4}\right)+\left(F_{4 y}^{q t}+P_{4}\right) l_{S 4} \cos \left(\varphi_{4}+\alpha_{4}\right) \\
F_{5 x}^{q t} \\
F_{5 y}^{q t}+P_{5} \\
M_{5}^{q t}-F_{5 x}^{q t} l_{S 5} \sin \left(\varphi_{5}+\alpha_{5}\right)+\left(F_{5 y}^{q t}+P_{5}\right) l_{S 5} \cos \left(\varphi_{5}+\alpha_{5}\right) \\
F_{6 x}^{q t} \\
F_{6 y}^{q t}+P_{6} \\
M_{6}+M_{6}^{q t}-F_{6 x}^{q t} l_{S 6} \sin \left(\varphi_{6}+\alpha_{6}\right)+\left(F_{6 y}^{q t}+P_{6}\right) l_{S 6} \cos \left(\varphi_{6}+\alpha_{6}\right)
\end{array}\right],
$$

where

$$
\begin{aligned}
& x_{i}=l_{i} \cos \varphi_{i}, \quad y_{i}=l_{i} \sin \varphi_{i}, \quad(i=2, \ldots, 6) \\
& x_{35}=l_{35} \cos \left(\varphi_{3}+\beta_{3}\right), \quad y_{35}=l_{35} \sin \left(\varphi_{3}+\beta_{3}\right) .
\end{aligned}
$$

The values of $\delta_{(i, j) x}$ and $\delta_{(i, j) y}$ are determined by using (2.8) and (2.9), then all the required quantities can now easily calculated from (3.25).

\section{Numerical results and discussion}

The main results of this study can be observed with a specific six-link mechanism. The linkage kinematic and dynamic parameters are given in Table 1. The coefficient of friction $\mu_{(i, j)}^{\gamma}=0.15$ and the pin radius $r_{(i, j)}=25 \mathrm{~mm}$ are chosen for all the joints.

Table 1. Parameters of the six-link mechanism

\begin{tabular}{|c|c|c|c|c|c|c|}
\hline Link $\mathrm{i}$ & $l_{i}(\mathrm{~mm})$ & $\beta_{i}(\mathrm{grad})$ & $l_{S i}(\mathrm{~mm})$ & $\alpha_{i}(\mathrm{grad})$ & $m_{i}(\mathrm{~kg})$ & $J_{S i}\left(\mathrm{kgmm}^{2}\right)$ \\
\hline 1 & 308 & 18.2 & & & & \\
\hline 2 & 143 & & 54 & 12.8 & 34 & 36070 \\
\hline 3 & 251 & 10.6 & 164 & 5.3 & 45 & 120340 \\
\hline 4 & 315 & & 98 & 12.0 & 14 & 15420 \\
\hline 5 & 420 & & 321 & 4.3 & 13 & 22760 \\
\hline 6 & 270 & & 130 & 10.2 & 22 & 23560 \\
\hline
\end{tabular}

For comparison between the calculating results with friction and without friction we consider three cases: The crank rotates at constant speeds of 600 rotations-per-minute 
(rpm), $3000 \mathrm{rpm}$ and $4500 \mathrm{rpm}$. Fig. 3 and Fig. 4 show some results obtained for the case of high speed (case 2). The resultant reaction forces at joint $(1,2)$ between the ground link and the crank and joint $(4,1)$ of the mechanism without and with friction are displayed in Fig. 3. The driving torque is shown in Fig. 4.

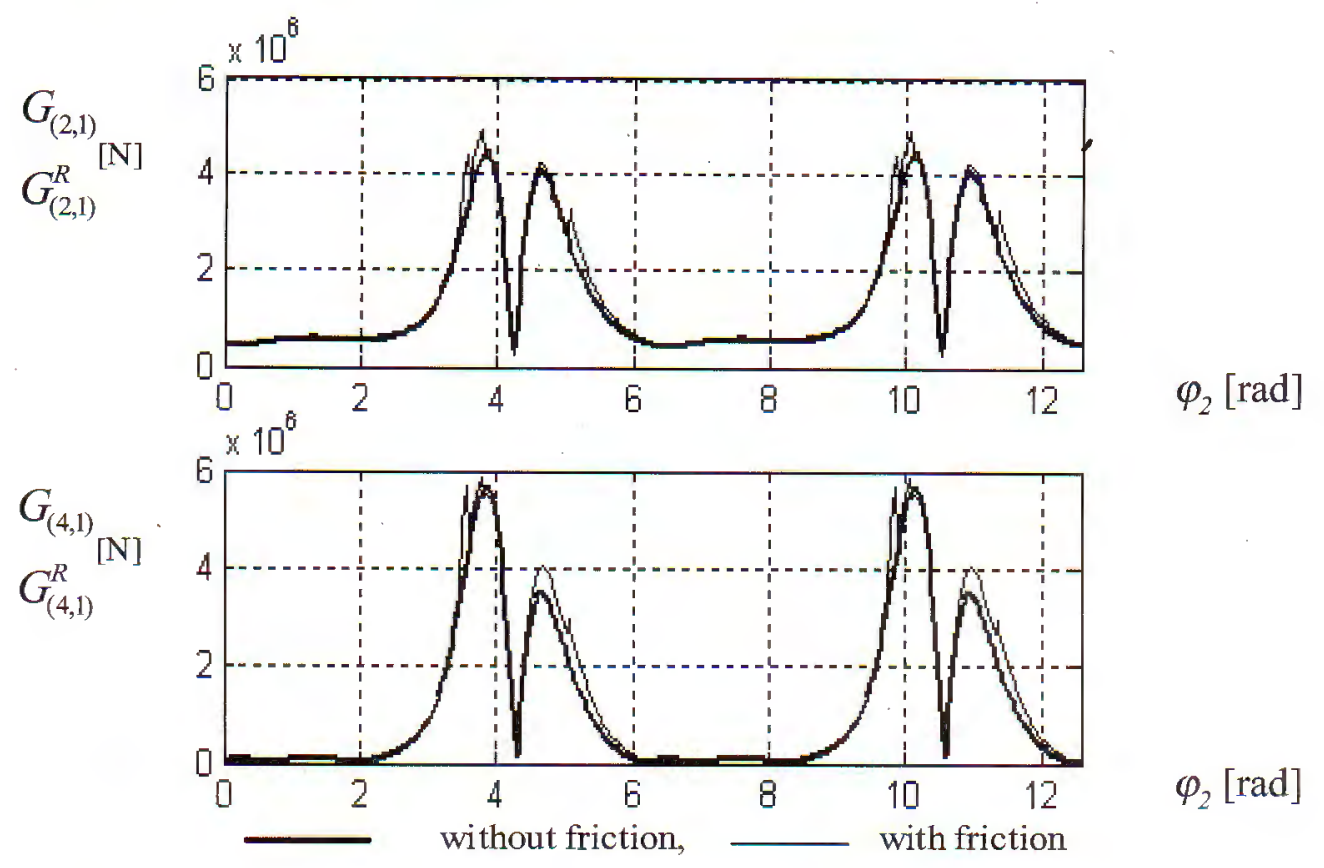

Fig. 3. Reaction forces at joint $(1,2)$ and joint $(4,1)$ at constant speed of $3000(\mathrm{rpm})$

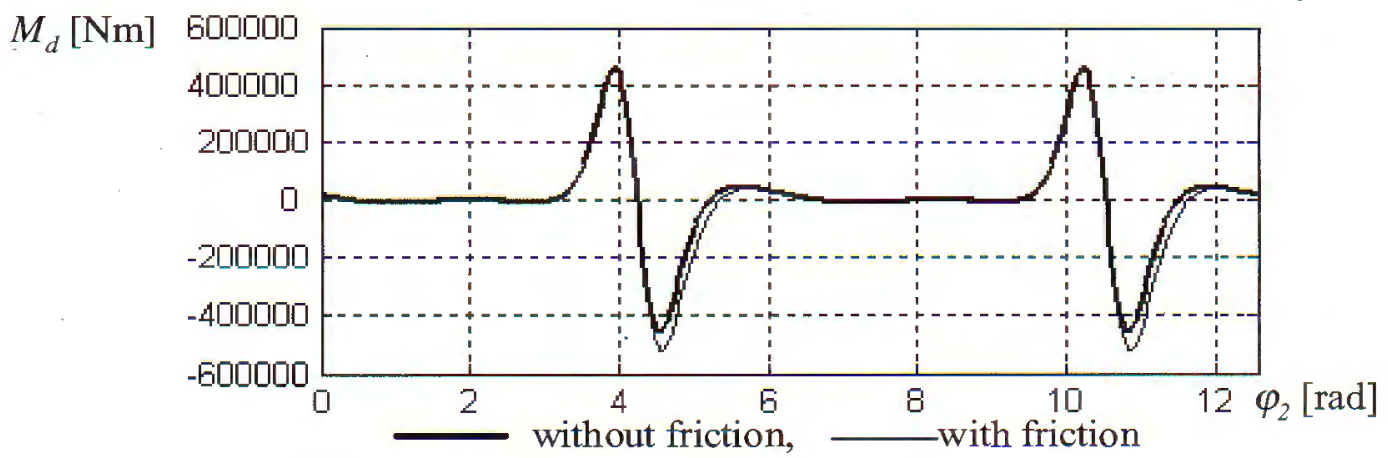

Fig. 4. Driving torque $M_{d}$ at constant speed of 3000 (rpm)

In order to evaluate influences of friction on the driving torque, the root-mean-square value (RMS-value) of this quantity is used. The difference between RMS-value of driving torques $M_{d}$ for the mechanism without friction and with friction increases proportionally with the increase of operational speed as shown in Table 2. For example, at the speed of $3000 \mathrm{rpm}$, the driving torques calculated under consideration of friction is $12.8 \%$ greater than one for the mechanism which is assumed to be free from friction. Therefore, the 
influences of friction at the joints of the mechanism may be very important for determining the driving torque required to generate a prescribed steady state motion at high speeds.

Table 2. RMS-value of the driving torque at various speeds of the crank

\begin{tabular}{|l|c|c|c|}
\hline Rotating speed $(\mathrm{rpm})$ & 600 & 3000 & 4500 \\
\hline RMS-value without friction $(\mathrm{kNm})$ & 6.4 & 159.48 & 359.25 \\
\hline RMS-value with friction $(\mathrm{kNm})$ & 7.2 & 179.89 & 405.19 \\
\hline
\end{tabular}

As indicated in section 2, the method used for the calculation in this example provides an approximation to the solution rather than the true value. The Taylor series of $G_{(i, j)}^{R}$ was truncated to the second term. Therefore, the truncation error $\varepsilon$ is introduced. The expression for the third term can be written in the form

$$
\begin{aligned}
\rho= & \frac{1}{2}\left\{\frac{\left(G_{(i, j) x}^{R}-G_{(i, j) x}\right)^{2}+\left(G_{(i, j) y}^{R}-G_{(i, j) y}\right)^{2}}{G_{(i, j)}}\right. \\
& \left.-\frac{\left[\left(G_{(i, j) x}^{R}-G_{(i, j) x}\right) G_{(i, j) x}+\left(G_{(i, j) y}^{R}-G_{(i, j) y}\right) G_{(i, j) y}\right]^{2}}{\left(G_{(i, j)}\right)^{3}}\right\} .
\end{aligned}
$$

Note that the value of $\rho$ is smaller than the second term in equation (2.5). In addition, the coefficient of $M_{(i, j)}^{R}$ in equations (3.12), (3.15), (3.18), (3.21), (3.24) can be evaluated as $1 / l_{i}$. From equation (2.1), the coefficient of $G_{(i, j)}^{R}$ is $r_{(i, j)} \mu_{(i, j)}^{\gamma} / l_{i}$. In engineering, the value of $\mu_{(i, j)}^{\gamma}$ is normally smaller than 0,3 and $r_{(i, j)} / l_{i}<0.5$. Hence, the truncation error $\varepsilon$ is smaller $0.15 \rho$. So, it can be concluded that the non-iterative approximate method provides a solution with sufficient accuracy for this problem.

\section{Conclusions}

The obtained results show that the friction at the joints has a great influence on the driving torque required for a prescribed steady state motion at high operational speeds of mechanisms. In addition, a significant difference between the curves of the joint reaction force without friction and ones with friction at high speed can be clearly observed. So, it can be seen that the frictional effect at the joints in such cases may be of considerable magnitude.

This publication is completed with the financial support from The Council for Natural Science of Vietnam.

\section{References}

1. Ghosh A., Mallik A. K., Theory of Mechanisms and Machines. Affiliated East-West Press, New Delhi, 1998.

2. Dresig H., Holzweissig, F., Maschinendynamik, Springer-Verlag, Berlin-Heidelberg, 2003. 
3. Funk W., Kong J. Y., Ein nicht-iteratives Naehrungsverfahren zur Ermittlung der Gelenkkraefte und Gleichgewichtsmomente von ebenen Koppelgetrieben unter Beruecksichtigung der Reibung. Archive of Applied Mechanics 64 (1994) 530-538.

4. Luck K., Modler K., Getriebetechnik, Springer-Verlag, Wien, 1990.

5. Dresig H., Vul'fson J. I., Dynamik der Mechanismen, Verlag Harri Deutsch / SpringerVerlag, Wien, 1989.

6. Nguyen Van Khang, Engineering Mechanics, Part I: Static and Kinematics. University and College Publisher, Hanoi, 2003 (in Vietnamese).

Received August 8, 2004

\section{PHÂN TÍCH ĐỘNG LỰC HỌC CO CẤU SÁU KHÂU PHẲNG KHI KỂ ĐẾN MA SÁT TẠI CÁC KHỚP}

Phân tích động lực học của cơ cấu phẳng khi kể đến ma sát tại các khớp là bài toán phức tạp do lực ma sát phụ thuộc phi tuyến vào thành phần phản lực tổng hợp giữa hai bề mặt tiếp xúc của khớp. Trong bài báo này, một phương pháp xấp xỉ không lặp đã được đề cập tới để xác định các phản lực khớp và mômen phát động của các cơ cấu phẳng. Nhờ phương pháp này, các phản lực động lực của cơ cấu sáu khâu phẳng đã được tính toán bằng hệ chương trình tính MATLAB. 